\title{
Renormalization of the Spin-Peierls Transition due to Phonon Dynamics
}

\author{
S. Trebst, N. Elstner and H. Monien \\ Physikalisches Institut, Universität Bonn, Nußallee 12, D-53115 Bonn, Germany
}

(November 12, 2017)

\begin{abstract}
We report results from a systematic strong-coupling expansion of a spin- $\frac{1}{2}$ Heisenberg chain coupled to Einstein phonons. In the non-adiabatic regime $(\hbar \Omega \approx J)$ this model is used to describe zero temperature properties of $\mathrm{CuGeO}_{3}$. The linked cluster expansion allows the determination of observables in the thermodynamic limit preserving the full lattice dynamics without a truncation of the phononic Hilbert space. In particular, the spin gap and the dispersion of the elementary triplet excitation are calculated up to $10^{\text {th }}$ order in a dimer expansion. The magnetic structure factor of the ground state is evaluated up to $6^{\text {th }}$ order. We show that the spin-phonon coupling leads to a renormalization of the elementary triplet dispersion. Surprisingly in the non-adiabatic regime a substantial renormalization of the spin gap only sets in at much larger couplings than those proposed for $\mathrm{CuGeO}_{3}$. The ground state magnetic correlations are found to be hardly effected by the spin-phonon coupling, but dominated by the frustrating magnetic interaction in the parameter regime relevant for $\mathrm{CuGeO}_{3}$.
\end{abstract}

PACS numbers: 75.10.Jm, 75.40.Mg, 75.50.Ee, 71.38.+i, 63.20.Kr

A renewed interest for the magnetic properties of one dimensional spin chains was created by the observation of a spin-Pejerls (SP) transition in the inorganic compound $\mathrm{CuGeO}_{3}$. The SP phase of $\mathrm{CuGeO}_{3}$ is characterized by an energy gap in the spin triplet excitation and a dimerization of the lattice along the chain direction. Experimentally, the nature of the SP transition was confirmed by inelastic neutron scattering (INS susceptibility and

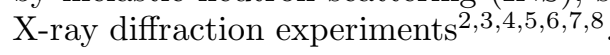

The SP transition is usually driven by the coupling of spins to the lattice. Previous theoretical work treated the spin-phonon coupling in terms of a static dimerization 6 . This approach does not allow the lattice to adjust to spin fluctuations and can only be expected to be valid in the adiabatic regime. In the case of $\mathrm{CuGeO}_{3}$ it has been shown that the magnetic energy scales given by the antiferromagnetic exchange integral $J$ and the phonon frequencies $\Omega$ are of the same order of magnitude $\Theta$. Thus, a realistic medel of $\mathrm{CuGeO}_{3}$ has to include dynamical phonons 10, 11, 12, 13, 14, 15. For technical reasons only very little is known about the experimental relevant regime with next-nearest noighbor coupling and intermediate phonon coupling 10.11.22. In this Letter we present numerically reliable results in the experimentally relevant regime of intermediate phonon frequencies including a frustrating next-nearest neighbor interaction.

We investigate a spin- $\frac{1}{2}$ Heisenberg chain coupled to dispersionless Einstein phonons with frequency $\Omega$

$$
\begin{aligned}
H=J \sum_{i}\left[\left(1+g\left(b_{i}^{\dagger}+b_{i}\right)\right) \vec{S}_{i} \cdot \vec{S}_{i+1}\right. & \left.+\alpha \vec{S}_{i} \cdot \vec{S}_{i+2}\right] \\
& +\Omega \sum_{i} b_{i}^{\dagger} b_{i}
\end{aligned}
$$

Here, the $b_{i}^{\dagger}$ and $b_{i}$ are the local phonon creation and annihilation operators respectively and $g$ is the spin-phonon coupling. The ratio of nearest to a frustrating nextnearest exchange coupling is given by $\alpha$.

There are two physically independent mechanisms in this model that can cause a dimerization. First, without spin-phonon coupling the frustrating next-nearest neighbor coupling parameterized by $\alpha$ drives a zero temperature quantum phase transition from a spin liquid to a dimerized phase at a critical value $\alpha_{c}$. A value of $\alpha_{c}=0.241$ was determined by numerical studies 18 . In the anti-adiabatic limit the phonons can be integrated out and the model can be mapped onto a system with a frustrating next-nearest neighbor interaction 16 . For $\alpha=0$ a phase transition from a gapless spin fluid to a gapped dimerized phase occurs at a non-zero value of the spin-phonon coupling. These results were confirmed to hold in the non-agiabatic and adiabatic regimes by numerical studies 13, 26, 14. This in particular is a feature of the dynamical model, since the static model exhibits a dimerization for all non-vanishing couplings 6 .

Our numprical approach is based on a linked cluster expansion 1920 which allows to evaluate physical observables that are additive when the system separates into disconnected parts. It is an inherent feature of cluster expansions that physical observables are evaluated in the thermodynamic limit. We calculated expansions for the ground state energy, $E_{0}$, the dispersion of the elementary spin triplet excitation $E_{T S}(\mathbf{q})$ including the spin gap $\Delta$ and the structure factor $S(\mathbf{q})$. The resulting series were analyzed using Padé extrapolation techniques.

In order to perform a dimer expansion of the Hamiltonian (1) we divide the Hamiltonian as 


$$
H=H_{0}+H_{1}
$$

where we shift the phonon operators

$$
b_{i}=\tilde{b}_{i}-\frac{g}{\Omega} \vec{S}_{i} \cdot \vec{S}_{i+1}
$$

which yields an exactly solvable Hamiltonian $H_{0}$

$$
\begin{aligned}
H_{0}=J \sum_{i}\left[\vec{S}_{i} \cdot \vec{S}_{i+1}-\frac{g^{2}}{\Omega}\left(\vec{S}_{i} \cdot \vec{S}_{i+1}\right)^{2}\right] & +\Omega \sum_{i} \tilde{b}_{i}^{\dagger} \tilde{b}_{i} \\
& +\Omega \sum_{j} b_{j}^{\dagger} b_{j}
\end{aligned}
$$

Here the index $i$ runs over the strong dimer bonds while the index $j$ runs over the weak bonds between the dimers.

The eigenstates of the Hamiltonian $H_{0}$ are described as a product of spin and phonon states. The spin states are products of local singlet and triplet dimer states. As a consequence of the transformation (2) the phonon states on the strong dimer bonds are coherent states given by

$$
\left|\tilde{0}_{s / t}\right\rangle=\exp \left(-\eta_{s / t}^{2} / 2\right) \cdot \exp \left(\eta_{s / t} b^{\dagger}\right)|0\rangle
$$

Here $\eta_{s}=3 g / 4 \Omega$ corresponds to a spin singlet state and $\eta_{t}=-g / 4 \Omega$ corresponds to a spin triplet state. The weak bonds between the dimers are occupied by $n_{j}$ quantum phonons, where $n_{j}=b_{j}^{\dagger} b_{j}$.

The perturbing Hamiltonian $H_{1}$ is then given by

$$
H_{1}=\lambda J \sum_{j}\left[\left(1+g\left(b_{j}^{\dagger}+b_{j}\right)\right) \vec{S}_{j} \cdot \vec{S}_{j+1}+\alpha \vec{S}_{j} \cdot \vec{S}_{j+2}\right]
$$

where the index $j$ runs over the weak bonds between the dimers. The expansion parameter $\lambda$ describes the ratio of the spin-spin coupling of the weak and strong bonds. The expansion is systematic in $\lambda$ while the spin-phonon coupling $g$ and the next-nearest neighbor interaction $\alpha$ are fixed for each evaluation of a series.

The total Hilbert-space of the Hamiltonian (11) is the tensorial product of the space of the spin configurations and the phononic space. While there is a finite set of spin configurations for every finite cluster, the Hilbert space associated with the phonons is infinite even for a finite cluster.

As a starting point for series expansions we use an initial wavefunction that describes a dimerized spin phase of spin singlets with a macroscopic occupation of phonon states on the dimer bonds and no quantum phonons on the bonds between the dimers. Performing a series expansion, only a limited number of quantum phonons is created on the weak bonds between the dimers. The macroscopic occupation numbers of the phonon states on the strong dimer bonds are tied to the fluctuations of the underlying spin states. Thus, the overall phononic Hilbert space is finite for a series expansion tofinite order. In contrast to previous theoretical work 10.11 we are not forced to truncate the phononic Hilbert space.
Series expansions were performed up to $10^{\text {th }}$ order with ten contributing clusters. The largest cluster contains eleven dimers or an equivalent of 22 spins. The Hilbert space for this cluster has 9,156,836 contributing states. Calculations were performed on local workstations.

As a closing remark to these technical aspects it is stressed again that our calculations do not require any finite size scalings.

The antiferromagnetic exchange integral $J$ and the next-nearest neighbor exchange integral $J^{\prime}$ are determined from fits to magnetic susceptibility data. The parameter set $J=160 K$ and $\alpha=0.36$ was estimated for $\mathrm{CuGeO}_{3}$ ]. The frequencies of the Peierls-active phonon modes were shown to be of the order of the magnetic energy scales $\mathrm{\theta}, \Omega_{1}=1 \mathrm{~J}$ and $\Omega_{2}=\mathrm{T}^{2} \mathrm{~J}$. Recently, the corresponding coupling constants 17 were proposed to be $g_{1}=-0.096$ and $g_{2}=0.16$. We will investigate our model with these parameters. To make contact to previous numerical studies 11 a third parameter set with $\Omega_{3}=0.3 \mathrm{~J}$ and $g_{3}=0.11$ is taken into account.

For the spin- $\frac{1}{2}$ Heisenberg chain with static dimerization $\delta$ several studies of the gap dependency on the static dimerization were performed. The corresponding Hamiltonian is given by

$$
\left.H^{\prime}=J_{0} \sum_{i}\left[\left(1+(-1)^{i} \delta\right)\right) \vec{S}_{i} \cdot \vec{S}_{i+1}+\alpha_{0} \vec{S}_{i} \cdot \vec{S}_{i+2}\right]
$$

The parameters of this model are related to those of Hamiltonian (11) by

$$
\begin{aligned}
& J=J_{0}(1+\delta) \\
& \lambda=(1-\delta) /(1+\delta) \\
& \alpha=\alpha_{0} /(1+\delta) .
\end{aligned}
$$

At the critical point the triplet gap $\Delta$ is known to show a $\Delta(\delta) \propto \delta^{2 / 3}$ dependency 6 . For supercritical frustration $\left(\alpha>\alpha_{c}\right)$ a dependency $\Delta(\delta)-\Delta(0) \propto \delta^{2 / 3}$ was recently proposed by Uhrig et. al. 21 .

In order to recover the original Hamiltonian (11) the evaluated series have to be analyzed at $\lambda=1$. We have performed two types of Padé approximations. First, motivated by the proposed supercritical dependency, we evaluate biased Padés fixing the exponent to be $2 / 3$. Second, assuming a power law dependency without restricting the exponent, we evaluate Dlog Padé approximants22 and reintegrated the obtained series to evaluate the gap.

For small supercritical values of $\alpha$ and small coupling constants $g$ both Padé approximants show well matching results. Varying the degree of numerator and denominator polynoms does not substantially change the resulting values justifying both approaches. For values of $\alpha$ and $g$ far away from critical points the biased Padé approximants become less reliable while the Dlog Padé approximants still give stable results as can be seen in table 四.

The inset in Fig. 11 shows the gap in units of $J_{0}$ versus the frustration $\alpha_{0}$. The filled symbols are obtained by 


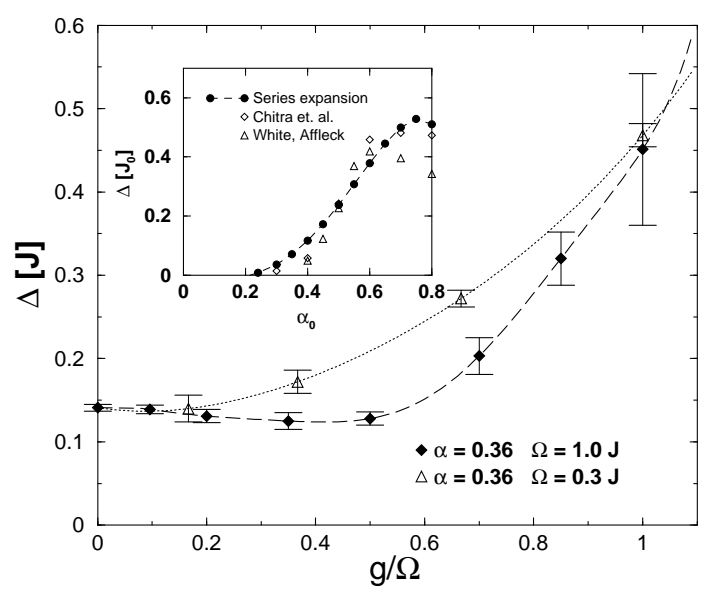

FIG. 1. Gap versus spin-phonon coupling. The dashed line is a polynomial fit to the data. The inset shows the gap versus the frustration in the static model, filled symbols are obtained by evaluation of the biased [5,5]-Padé approximant. The open symbols show DMRG data 23 .

evaluation of the biased [5,5]-Padé approximant. The open symbols show DMRG data23. Our series results are in full agreement with those obtained by the perturbative approach using flow equations 2 . Good agreement is also found with previous DMRG results.

Fig. 1 shows the gap in units of $J$ for a frustration of $\alpha=0.36$ for various spin-phonon couplings and phonon frequencies. The error bars represent the deviation of the accepted Dlog Padé approximants. The dashed lines are polynomial fits to the data. For the parameter set $\alpha=0.36, \Omega_{3}=0.3 \mathrm{~J}$, and $g_{3}=0.11$ we find a triplet gap $\Delta=(0.172 \pm 0.014) J$, which is in good agreement with previous exact diagonalization extrapolations for the $\pi$ phonon mode11. For the parameters relevant to $\mathrm{CuGeO}_{3}$ our results are below the xperimentally obtained value

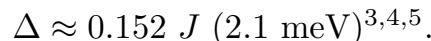

In the case of a nearly adiabatic phonon frequency $\Omega=0.3 J$ the gap $\Delta$ monotonically increases with higher spin-phonon couplings. For the non-adiabatic phonon frequency $\Omega=1 J$ the gap shows a gentle downward slope. This indicates a suppression of the frustration for small phonon frequencies due to a stronger renormalization of the nearest neighbor spin inferaction than the next-nearest neighbor spin interaction ${ }^{12}$. For higher couplings this is compensated by an overcritical coupling.

The dispersion of the elementary triplet excitation is shown in Fig. 2. The series expansion method is based on Rayleigh-Schrödinger perturbation theory and is therefore supposed to produce well converging results if the contributing states are clearly separated by an energy gap. In the region $|\mathbf{q}|>0.4 \pi$ the series converges very fast. For smaller momenta Dlog Padé approximants are required to obtain numerically reliable results.

It turns out that the two parameter sets relevant for $\mathrm{CuGeO}_{3}$ give nearly matching dispersion relations. The

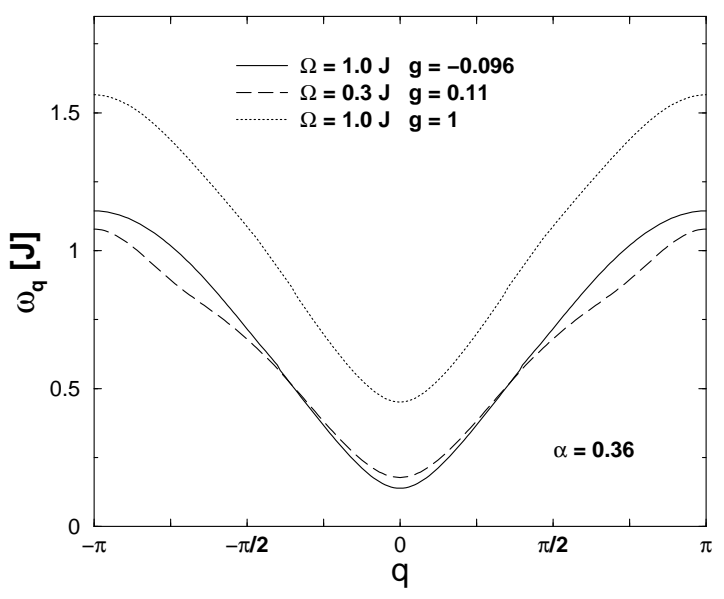

FIG. 2. Dispersion of the elementary triplet excitation evaluated by a dimer expansion up to $10^{\text {th }}$ order for different phonon frequencies and spin-phonon coupling constants. For each momentum the series were analyzed using Dlog Padé approximants.

maxima of the dispersion relation at $\mathbf{q}= \pm \pi$ are suppressed in comparison with the known value $\omega(\mathbf{q}=$ $\pm \pi)=\pi / 2$ for the unperturbed Heisenberg chain which is mainly due to the next-nearest neighbor magnetic interaction.

The Peierls ordering structure of the ground state is accompanied by short range magnetic order. Thus, we evaluate the following real-space magnetic correlation function

$$
S_{j}=\left\langle\vec{N}_{0} \cdot \vec{N}_{2 j+2}\right\rangle
$$

where $\vec{N}_{j}=\vec{S}_{j}-\vec{S}_{j+1}$ is the sublattice magnetization and $\vec{S}_{j}$ denotes the spin on site $j$. Technically, this is done by formally adding a source term

$$
H_{2}=h \sum_{j} \vec{N}_{0} \cdot \vec{N}_{2 j+2}
$$

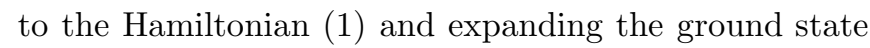
energy to linear order in $h$. Differentiating with respect to $h$ will give the strong coupling expansion for the correlation function. The determined structure factors are well converged. The results presented in the following are obtained by direct evaluation of the series without using Padé approximants.

The Fourier transform

$$
S(\mathbf{q})=\sum_{j} e^{i \mathbf{q} \cdot \mathbf{r}_{j}} S_{j}
$$

is shown in Fig. 3. Parameters are chosen in the gapped phase where the structure factor shows a Lorentzian shape which obviously differs from the logarithmic divergence of the isotropic Heisenberg model at $S(\mathbf{q}=0)$. In the case of weak spin-phonon coupling as given in 


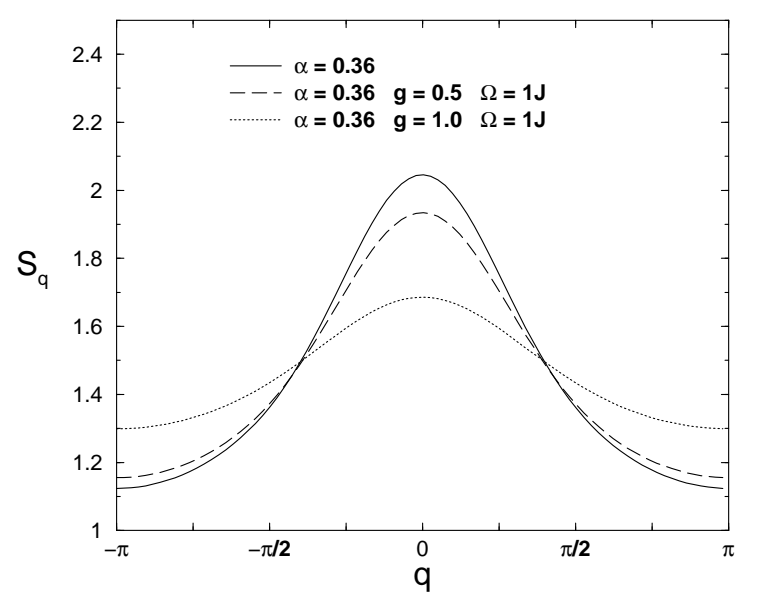

FIG. 3. Structure factor $S(\mathbf{q})$ versus momentum $\mathbf{q}$ in a sixth order calculation.

$\mathrm{CuGeO}_{3}$ this is mostly due to the next-nearest neighbor interaction. The evaluated series with next-nearest neighbor interaction and the series with additional spinphonon coupling cannot be reasonably distinguished. For stronger couplings Fig. 3 shows that the spin-phonon interaction leads to a further diminishing of the maximum at $\mathbf{q}=0$ and a decrease of the spin correlation length.

In conclusion, series expansion techniques were applied to investigate zero temperature properties of a spin- $\frac{1}{2}$ Heisenberg chain coupled to Einstein phonons which in the non-adiabatic limit serves as a model for $\mathrm{CuGeO}_{3}$. We evaluated the dispersion of the elementary triplet excitation and demonstrated that the spin-phonon coupling leads to a renormalization of the dispersion. The dependency of the triplet gap on the spin-phonon coupling shows a non-monotonous behavior in the non-adiabatic regime. For the parameters relevant to $\mathrm{CuGeO}_{3}$ no substantial renormalization of the triplet gap is found. The magnetic ordering of the ground state was determined by calculation of the spin structure factor. It was found that the next-nearest neighbor interaction dominates the spin correlations in the case of $\mathrm{CuGeO}_{3}$.

We acknowledge helpful discussions on this problem with G. S. Uhrig, S. Eggert, R. Werner and C. Gros.

${ }^{1}$ M. Hase, I. Terasaki, and K. Uchinokura, Phys. Rev. Lett. 70, 3651 (1993).

2 J. P. Pouget, L. P. Regnault, M. Aïn, B. Hennion, J. P. Renard, P. Veillet G. Dhalenne, and A. Revcolevschi, Phys. Rev. Lett. 72, 4037 (1994).

${ }^{3}$ Q. J. Harris, Q. Feng, R. J. Birgeneau, K. Hirota, K. Kakurai, J. E. Lorenzo, G. Shirane, M. Hase, K. Uchinokura, H. Kojima, I. Tanaka, and Y. Shibuya, Phys. Rev. B. 50,
TABLE I. $[n / m]$ Dlog Padé approximants to the series for the energy gap $\Delta$ for a frustration $\alpha=0.36$ and a phonon frequency $\Omega=1 \mathrm{~J}$. Values are given in units of $J$.

\begin{tabular}{|c|c|c|c|c|}
\hline & {$[(\mathrm{n}-2) / \mathrm{n}]$} & {$[(\mathrm{n}-1) / \mathrm{n}]$} & {$[\mathrm{n} / \mathrm{n}]$} & {$[(\mathrm{n}+1) / \mathrm{n}]$} \\
\hline \multicolumn{5}{|c|}{$g=0$} \\
\hline$n=4$ & - & 0.136 & 0.145 & 0.099 \\
\hline $\mathrm{n}=5$ & 0.145 & 0.138 & & \\
\hline \multicolumn{5}{|c|}{$g=0.096$} \\
\hline$n=4$ & - & 0.133 & 0.143 & 0.098 \\
\hline $\mathrm{n}=5$ & 0.143 & 0.135 & & \\
\hline \multicolumn{5}{|c|}{$g=0.2$} \\
\hline $\mathrm{n}=4$ & - & 0.120 & 0.139 & 0.096 \\
\hline $\mathrm{n}=5$ & 0.140 & 0.126 & & \\
\hline \multicolumn{5}{|c|}{$g=0.5$} \\
\hline $\mathrm{n}=4$ & - & - & 0.125 & 0.121 \\
\hline $\mathrm{n}=5$ & 0.142 & 0.124 & & \\
\hline \multicolumn{5}{|c|}{$g=1.0$} \\
\hline$n=4$ & - & 0.277 & 0.461 & 0.503 \\
\hline $\mathrm{n}=5$ & 0.474 & 0.540 & & \\
\hline
\end{tabular}

12606 (1994).

${ }^{4}$ M. Nishi, O. Fujita, and J. Akimitsu, Phys. Rev. B. 50, R6508 (1994).

${ }^{5}$ L. P. Regnault, M. Aïn, B. Hennion, G. Dhalenne, and A. Revcolevschi, Phys. Rev. B. 53, 5579 (1996).

${ }^{6}$ M. Braden, B. Hennion, W. Reichardt, G. Dhalenne, and A. Revcolevschi, Phys. Rev. Lett. 80, 3634 (1998).

${ }^{7}$ J. Riera and A. Dobry, Phys. Rev. B. 51, 16098 (1995).

${ }^{8}$ K. Fabricius, A. Klümper, U. Löw, B.Büchner, T. Lorenz, G. Dhalenne, and A. Revcolevschi, Phys. Rev. B. 57, 1102 (1998).

${ }^{9}$ M. C. Cross and D. S. Fisher, Phys. Rev. B. 19, 402 (1979).

${ }^{10}$ D. Augier and D. Poilblanc, Eur. Phys. J. B 1, 19 (1998).

${ }^{11}$ G. Wellein, H. Fehske, and A. P. Kampf, Phys. Rev. Lett. 81, 3956 (1998).

12 A. Weiße, G. Wellein, and H. Fehske, cond-mat/9901262, to appear in Phys. Rev. B. 60 (1999).

${ }^{13}$ R. J. Bursill, R. H. McKenzie, and C. J. Hamer, Phys. Rev. Lett. 83, 408 (1999).

${ }^{14}$ A. W. Sandvik and D. K. Campbell, Phys. Rev. Lett. 83, 195 (1999).

${ }^{15}$ R. W. Kühne and U. Löw, cond-mat/9905337.

${ }^{16}$ G. S. Uhrig, Phys. Rev. B. 57, R14004 (1998)

${ }^{17}$ R. Werner, C. Gros, and M. Braden, cond-mat/9810038.

18 S. Eggert, Phys. Rev. B. 54, R9612 (1996).

19 M.P. Gelfand, R.R.P. Singh, and D.A. Huse, J. Stat. Phys. 59, 1093 (1990).

${ }^{20}$ M. P. Gelfand, Sol. Stat. Com. 98, 11 (1996).

${ }^{21}$ G. S. Uhrig, F. Schönfeld, M. Laukamp, and E. Dagotto, Eur. Phys. J. B. 7, 67 (1999).

${ }^{22}$ G. A. Baker, Jr., Quantitative theory of critical phenomena, Academic Press, Boston, 1990.

${ }^{23}$ R. Chitra, S. Pati, H. R. Krishnamurthy, D. Sen, and S. Ramasesha, Phys. Rev. B. 52, 6581 (1995). S. R. White and I. Affleck, Phys. Rev. B. 54, 9862 (1996).

${ }^{24}$ C. Knetter and G. S. Uhrig, cond-mat/9906243 to appear in Eur. Phys. J. B. 